\title{
A Case for Lorentzian Relativity
}

\author{
Daniel Shanahan
}

Received: 4 February 2013 / Revised: 3 November 2013 / Accepted: 11 November 2013

/ Published online: 1 January 2014

(C) The Author(s) 2013. This article is published with open access at Springerlink.com

\begin{abstract}
The Lorentz transformation (LT) is explained by changes occurring in the wave characteristics of matter as it changes inertial frame. This explanation is akin to that favoured by Lorentz, but informed by later insights, due primarily to de Broglie, regarding the underlying unity of matter and radiation. To show the nature of these changes, a massive particle is modelled as a standing wave in three dimensions. As the particle moves, the standing wave becomes a travelling wave having two factors. One is a carrier wave displaying the dilated frequency and contracted ellipsoidal form described by the LT, while the other (identified as the de Broglie wave) is a modulation defining the dephasing of the carrier wave (and thus the failure of simultaneity) in the direction of travel. The superluminality of the de Broglie wave is thus explained, as are several other mysterious features of the optical behaviour of matter, including the physical meaning of the Schrödinger equation and the relevance to scattering processes of the de Broglie wave vector. Consideration is given to what this Lorentzian approach to relativity might mean for the possible existence of a preferred frame and the origin of the observed Minkowski metric.
\end{abstract}

Keywords Special relativity - Lorentzian relativity - Covariance $\cdot$ de Broglie wave . Schrödinger equation

\section{Introduction}

For the student of physics, there comes a moment of intellectual pleasure as he or she realizes for the first time how changes of length, time and simultaneity conspire to

D. Shanahan $(\varangle)$

18 Sommersea Drive, Raby Bay, QLD 4163, Australia

e-mail: danjune@bigpond.net.au 
preserve the observed speed of light. Yet Einstein's theory [1] provides little understanding of how Nature has contrived this apparent intermingling of space and time.

The language of special relativity (SR) may leave the impression that the Lorentz transformation (LT) describes actual physical changes of space and time. Thus we have Minkowski's confident prediction that,

Henceforth, space by itself, and time by itself, are doomed to fade away into mere shadows and only a kind of union of the two will preserve an independent reality [2].

The impression that the LT involves some physical transmutation of "spacetime" might seem consistent with the change of that nature contemplated in general relativity (GR). But in GR a change in the metric affects in like manner all that occupies the region of space in question. In SR it is necessary to distinguish what actually changes from what is merely "observed"1 to change.

Consider two explorers, who we will call Buzz and Mary. They had been travelling, in separate space ships, side by side, in the same direction. But Buzz has veered away to explore a distant asteroid. Mary concludes from her knowledge of the LT that time must now be running more slowly for Buzz and that he and his ship have become foreshortened in the direction that Buzz is travelling relative to her. Buzz observes no such changes either in himself or in his ship. To Buzz, it is in Mary and her ship that these changes have occurred. Buzz is also aware that events that he might previously have regarded as simultaneous are no longer so.

No relevant physical change has occurred in Mary or her spacecraft. She has not accelerated. She is in the same inertial frame as before. Nor (ignoring gravitational effects, in this case negligible) has any actual, as distinct from observed, change occurred in the space through which the travellers are moving. To suggest otherwise would be to suppose that space is able to contract in one way for one particle and in a different way for another moving relatively to the first, albeit that the two (or at least their correspondingly contracted fields) could be occupying the very same piece of space. Even Buzz will have realized, as he observed the contraction of the constellations, that the stars were not in fact closing ranks around him.

A change of inertial frame has occurred for Buzz and his spacecraft. It must be this change that is the source of the changes that the two explorers are observing. It is not difficult to understand that Buzz's change of velocity may have caused a change in him that has affected how he is perceived by Mary. But it must also be the case, since nothing else has changed, that it is this same change in Buzz that has caused him to consider the (in fact unchanged) Mary in a different light.

Buzz will not sense that he has changed. After all everything in his inertial frame will have changed in like manner. Unlike the carousel rider who sees the fairground whirling about her, but is under no illusion as to what is really happening, Buzz has suffered relativistic changes in his vital processes, and lost the means of discernment. For Buzz the LT will describe very well his altered perspective. But it would be as

\footnotetext{
1 "Observed" is used here, in the sense conventional in SR, to denote not what is "seen" at a particular instant, but what the observer considers to have occurred at that instant. What is seen includes light from events that occurred progressively earlier the further they were away, an effect here irrelevant.
} 
inappropriate to explain length contraction, time dilation and loss of simultaneity as resulting from a physical transformation of space or spacetime as it would be to describe the rotation of an object in 3-space as a rotation of space rather than a rotation in space.

While one might wish to elevate the discussion by reference to differential manifolds, the spacetime continuum, or the Minkowski metric, the curious effects described by the LT must be explained by a change that occurs in matter as it suffers a "boost" from one inertial frame to another. Indeed the Minkowski metric should itself be seen as a kind of illusion, and as a consequence rather than the cause of this change in matter.

But to entertain these thoughts is to embark upon a process of reasoning, associated primarily with Lorentz, that became unfashionable ${ }^{2}$ following Einstein's famous paper of 1905 [1]. Lorentz had sought to explain the transformation that bears his name on the basis of changes that occur in matter as it changes velocity. This was, it is suggested, an idea before its time. We will consider in this paper how Lorentz's program might have proceeded if informed by later insights as to the underlying wave nature of matter, including ironically those of Einstein himself, but in particular that of de Broglie [9].

\section{Lorentz and Einstein}

Briefly first some history. The problem addressed by Lorentz and subsequently Einstein was the speed of light. This emerged as a constant in Maxwell's equations, but if as was generally supposed, light is wave-like, it seemed reasonable to assume that it must be carried by some medium (the "luminiferous aether") at a velocity characteristic of that medium. Thus its velocity relative to an observer should have varied with the motion of the observer through the medium. Experiments of increasing sophistication failed to reveal any trace of that variation.

Several explanations were put forward. It was proposed that the Earth must carry the local aether with it, but a more fruitful suggestion made independently by Fitzgerald [10] and Lorentz [11] was that objects moving through the aether must be somehow shortened along their direction of travel, thereby disguising relative changes in the velocity of light. It was supposed that intermolecular forces must be transmitted at the same velocity as electromagnetic waves, so that movement through the aether would influence the degree of attraction between molecules and thus the separation of those molecules.

To effect a reconciliation with Maxwell's equations, it was necessary to assume changes not only of length, but also of time, and thus the LT,

\footnotetext{
2 Described as "best forgotten except by historians" by Rindler [3, p. 11]. For views more consonant with those offered here, see Bell [4], Brown and Pooley [5], and in particular, Brown [6]. The pedagogical merits of this "constructive" approach to SR are also discussed in Miller [7], and Nelson [8].
} 


$$
\begin{aligned}
x^{\prime} & =\gamma(x-v t), \\
y^{\prime} & =y, \\
z^{\prime} & =z, \\
t^{\prime} & =\gamma\left(t-\frac{v x}{c^{2}}\right),
\end{aligned}
$$

where $c$ is the speed of light, $\gamma$ is the Lorentz factor,

$$
\left(1-\frac{v^{2}}{c^{2}}\right)^{-\frac{1}{2}}
$$

and the unprimed coordinates are for an event in the observer's rest frame, while the primed are for those in a frame moving in the $x$ direction at the velocity $v$.

The LT was already reasonably well known by 1905 . There had been significant contributions to its development, not only from Lorentz and Fitzgerald, but also by (among others) Heaviside, Larmor and Poincaré. It was Heaviside's [12] analysis of the disposition of fields accompanying a charged particle (the "Heaviside ellipsoid") that had suggested to FitzGerald the idea of length contraction. Larmor [13] had described an early form of the LT and discussed the necessity of time dilation. Poincaré [14] had recognized the relativity of simultaneity and had studied the group theoretic properties that form the basis for the covariance of the transformation.

But these "trailblazers" (Brown [6, Chap. 4]) appear to have missed in varying degrees the full significance of the transformation. ${ }^{3}$ It is not only particular phenomena, but all of Nature that changes for the accelerated observer. Lorentz struggled to explain how all aspects of matter could became transformed in equal measure, being discouraged by experimental reports that seemed to show that particles do not contract in the direction of travel (see Brown [6], p. 86). A wider view seems to have been noticed by Poincaré [14], who has been regarded by some as codiscoverer of SR (see, for instance, Zahar [16], and Reignier [15]). But it is not apparent that these earlier investigators saw the changes described by the LT as anything more than mathematical constructs. In his paper of 1905 [1], Einstein simply asserted that the velocity of light, and other properties of Nature, must appear the same for all uniformly moving observers, thereby effecting an immediate reconciliation between Maxwell's equations and classical mechanics.

In 1905, Einstein's approach may have been the only way forward. It was not until 1924, only a few years before the death of Lorentz, and well after that of Poincaré, that de Broglie [9] proposed that matter is also wavelike, an idea that might have suggested to Lorentz why molecules become transformed in the same degree as intermolecular forces. But as inadequate and ad hoc as Lorentz's suggestions may have seemed at the time, he at least had sought an underlying physical basis for the transformation. Lorentz commented, presumably with some chagrin, that,

\footnotetext{
3 There are differing opinions as to who knew what in 1905. For an interesting sampling of conflicting views on priority (Einstein, Lorentz or Poincaré?) see the preamble to Reignier [15].
} 
Einstein simply postulates what we have deduced, with some difficulty, and not altogether satisfactorily, from the fundamental equations of the electromagnetic field [17].

In what follows, the distinction drawn will be between Einstein's SR (ESR) and what we will call Lorentzian SR (LSR). This is not to diminish the contributions of others, but it was Lorentz in particular who sought to explain SR from underlying physical processes, as will be the objective below. Once the form of the LT was known, all else in SR then followed, including the composition of velocities, the group theoretic properties of the transformation, and the invariance of Maxwell's equations. It may be argued that with these refinements (largely due to Einstein and Poincaré), ESR and LSR are essentially equivalent. They cannot be distinguished, mathematically or empirically, through the privileged frame that was supposed by Lorentz, but declared "superfluous" by Einstein [1]. It would seem that any such frame is rendered undetectable by the covariance of the LT. Nor can ESR and LSR be distinguished by supposing that in ESR, though not in LSR, the LT describes a transformation of spacetime. As we have seen, the LT must be explained in either case by changes occurring in matter as it is accelerated from one inertial frame to another.

Historically, the two approaches are distinguished by the assumptions made by Einstein in justifying the LT. His confident assertion of these "postulates" gave impetus to the recognition and development of SR. But by raising "to the status of a postulate", the conjecture that Nature displays "no properties corresponding to the idea of absolute rest", and introducing the further postulate that the speed of light must be the same for all observers [1], Einstein avoided all necessity of explaining how Nature has arranged these matters. He left the impression that there was nothing more that needed explaining.

Einstein's postulates presuppose that everything in Nature transforms in the same degree. Accepting that this is so, we should suspect the existence of some unifying feature common to matter and radiation that ensures that it is so. If it had been possible to see the LT as a transformation of space carrying with it all within space, this would have explained very nicely the all-encompassing generality of the transformation. Having dismissed that possibility, we will proceed now on a different tack.

\section{The Scheme of the Paper}

It will be supposed (taking our cue from de Broglie [9]) that radiation and matter transform in like manner because they are constituted in like manner from similar wave-like influences. By modelling a massive particle as a standing wave in three dimensions, it will be shown in the next section (Sect. 4) that the changes in length, time and simultaneity described by the LT are the immediate consequences of changes in the wave structure of the particle as it changes inertial frame.

As discussed in Sect. 5, a compelling advantage of this treatment will be the emergence of the otherwise mysterious de Broglie wave, not as the independent wave generally supposed, but as a modulation defining the dephasing of the underlying wave structure in the direction of travel. This conception of the de Broglie wave is 
not itself new. Such a modulation may be discerned in a toy model described by de Broglie [9] himself in his famous doctoral thesis of 1924. It has been discussed in various contexts on several occasions since [18-22]. However, it will be shown here that this interpretation of the wave explains several curious features of the wave-like behaviour of matter. It avoids the anomalous nature of the superluminality of the wave, it makes sense of the role of the wave in quantization and in the Schrödinger equation; and it explains the relevance of the de Broglie wave number to the optical properties of massive particles. Its primary significance to this paper is that the dephasing defined by the modulation is the measure of the failure of simultaneity that is perhaps the most counter-intuitive aspect of SR.

In Sect. 6, we will consider the sufficiency of the model particle introduced in Sect. 4. It will be shown that this test particle exhibits, not so much the properties of all matter, but more relevantly, those implicitly assumed by ESR. In Sect. 7, consideration will be given to the possible existence of a preferred frame, that is to say, a frame in which the speed of light is not simply observed to be, but in fact is, the same in all directions. The position will be taken that the fixed rate at which photons are observed to propagate through space suggests very strongly, if not a light-carrying medium, then at least the preferred frame favoured by Lorentz.

In Sect. 8, it will be argued that "Lorentz invariance" - the invariance of the laws of physics to all inertial observers-is itself a consequence of the wave-like characteristics of matter. A brief summary in Sect. 9 will conclude the paper.

\section{A Model Particle}

In 1905, Einstein took as his yardstick, the rigid rod, avoiding consideration of underlying structure [1]. However a massive particle has, from the Planck-Einstein relation,

$$
E=\hbar \omega_{E}=\hbar \gamma \omega_{o}
$$

an associated frequency $\omega_{E}$, and from the de Broglie relation,

$$
p=\hbar \kappa_{d B}=\hbar \gamma \omega_{o} \frac{v}{c}
$$

a wave number $\kappa_{d B}$ (the de Broglie wave number), where $E$ and $p$ are respectively the energy and momentum of the particle, $\hbar$ is the reduced Planck's constant, and $\omega_{o}$ is the natural or characteristic frequency of the particle at rest.

Wave number $\kappa_{d B}$ and frequency $\omega_{E}$ define the wave that de Broglie referred to as a pilot wave, and that we now know as the de Broglie or matter wave. Considered as an independent wave it is anomalous. It has in free space the form of a transverse plane wave,

$$
e^{i \hbar\left(\omega_{E} t-\kappa_{d B} z\right)}
$$

of superluminal velocity $c^{2} / v$. What is also curious (though a pointer to the true nature of this wave) is that its speed increases as the particle slows, becoming infinite as the particle comes to rest. 
In its rest frame, the particle retains nonetheless the frequency $\omega_{o}$, which we will assume is not a fictitious or merely internal frequency, but the measure of some oscillatory disturbance communicated through space at velocity $c$. From this assumption we model a massive particle in its rest frame by the simple symmetrical waveform,

$$
\Psi(\mathbf{r}, t)=|\mathbf{r}|^{-1}\left[e^{i\left(\omega_{o} t-\kappa_{o} \cdot \mathbf{r}\right)}-e^{i\left(\omega_{o} t+\kappa_{o} \cdot \mathbf{r}\right)}\right] / 2,
$$

or, taking real parts,

$$
\Psi(\mathbf{r}, t)=|\mathbf{r}|^{-1} \sin \kappa_{o} \cdot \mathbf{r} \cos \omega_{o} t,
$$

which is a spherical standing wave centred at $\mathbf{r}=0$ and comprising incoming and outgoing waves of velocity $c$, where,

$$
\frac{\omega_{o}}{\kappa_{o}}=c,
$$

so that $\kappa_{o}$ is not here the de Broglie wave number but the wave number that must be associated with a wave of frequency $\omega_{o}$ and velocity $c .{ }^{4}$

Model particle (4) is depicted (in two dimensions) in Fig. 1a. This particular structure is evidently unphysical because of the singularity at $r=0$. It is nonetheless the simplest expression of the assumption, essential to ESR, that influences moving to and from (and indeed through) a particle do so at the speed of light. We will consider this model further in Sect. 6. We now investigate how this particle must change if it is to acquire a velocity $v$ in the frame of the laboratory, when subject to the constraint that its constituent influences (rays) must retain the velocity $c$ with respect to that frame. (We are thus assuming for the moment that the inertial frame in which this particle has the form (4), which we have called the laboratory frame, is at the same time the preferred or privileged frame supposed by Lorentz. To simplify matters further the amplitude $|\mathbf{r}|^{-1}$ is omitted in what follows).

What we require now is not a standing wave but a travelling wave. Its form can be established in either of two ways, and it will be instructive to consider both. The first is by construction, and we begin by considering rays, directed forward and rearward along the direction of travel, which we will take to be the positive $x$-direction. At rest in the preferred (laboratory) frame, the composition of these rays results in the one-dimensional standing wave

$$
\Psi(x, t)=\left[e^{i\left(\omega_{o} t-\kappa_{o} x\right)}-e^{i\left(\omega_{o} t+\kappa_{o} x\right)}\right] / 2,
$$

or, taking real parts,

$$
\Psi(x, t)=\sin \kappa_{o} x \cos \omega_{o} t
$$

This standing wave becomes a travelling wave of velocity $v$ if the wave characteristics of the rays directed forward and rearward (which we now label 1 and 2 respectively) become,

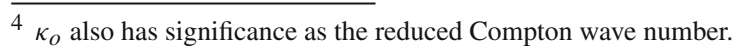


Fig. 1 A model particle is represented a as a spherical standing wave and $\mathbf{b}$ as a relativistically contracted carrier wave of velocity $v$, subject to a modulation (the de Broglie wave) of superluminal velocity $c^{2} / v$. The ellipses in $\mathbf{b}$ represent the wave fronts of the carrier wave and the vertical lines those of the de Broglie modulation (a)

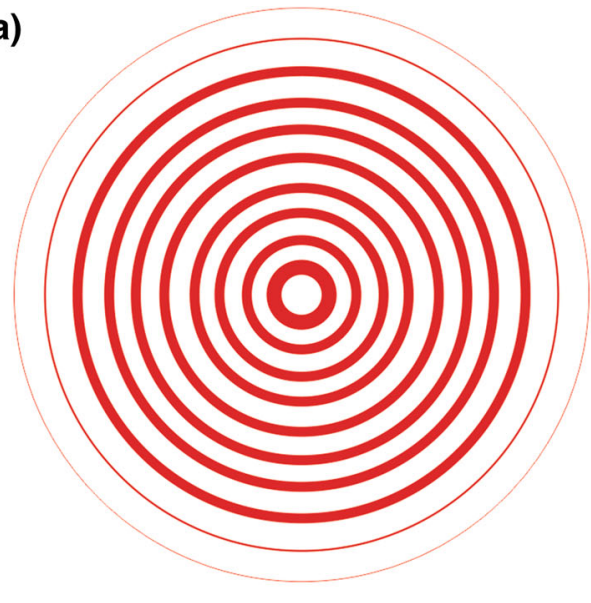

(b)

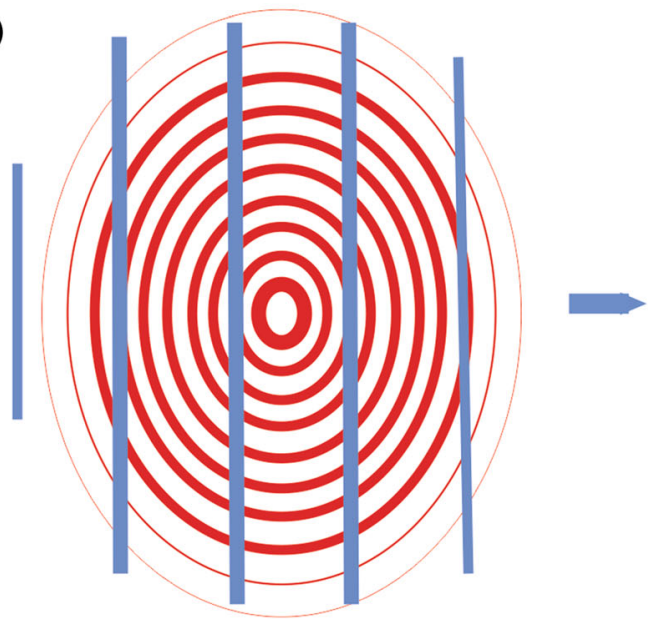

$$
\begin{aligned}
& \omega_{1}=\gamma \omega_{0}\left(1+\frac{v}{c}\right), \quad \omega_{2}=\gamma \omega_{0}\left(1-\frac{v}{c}\right), \\
& \kappa_{1}=\gamma \kappa_{0}\left(1+\frac{v}{c}\right), \quad \kappa_{2}=\gamma \kappa_{0}\left(1-\frac{v}{c}\right),
\end{aligned}
$$

where $\gamma$ is again the Lorentz factor (1), and since,

$$
\frac{\omega_{1}}{\kappa_{1}}=\frac{\omega_{2}}{\kappa_{2}}=\frac{\omega_{0}}{\kappa_{0}}=c,
$$

both rays retain as required the velocity $c$ with respect to the preferred frame. We then have by composition, 


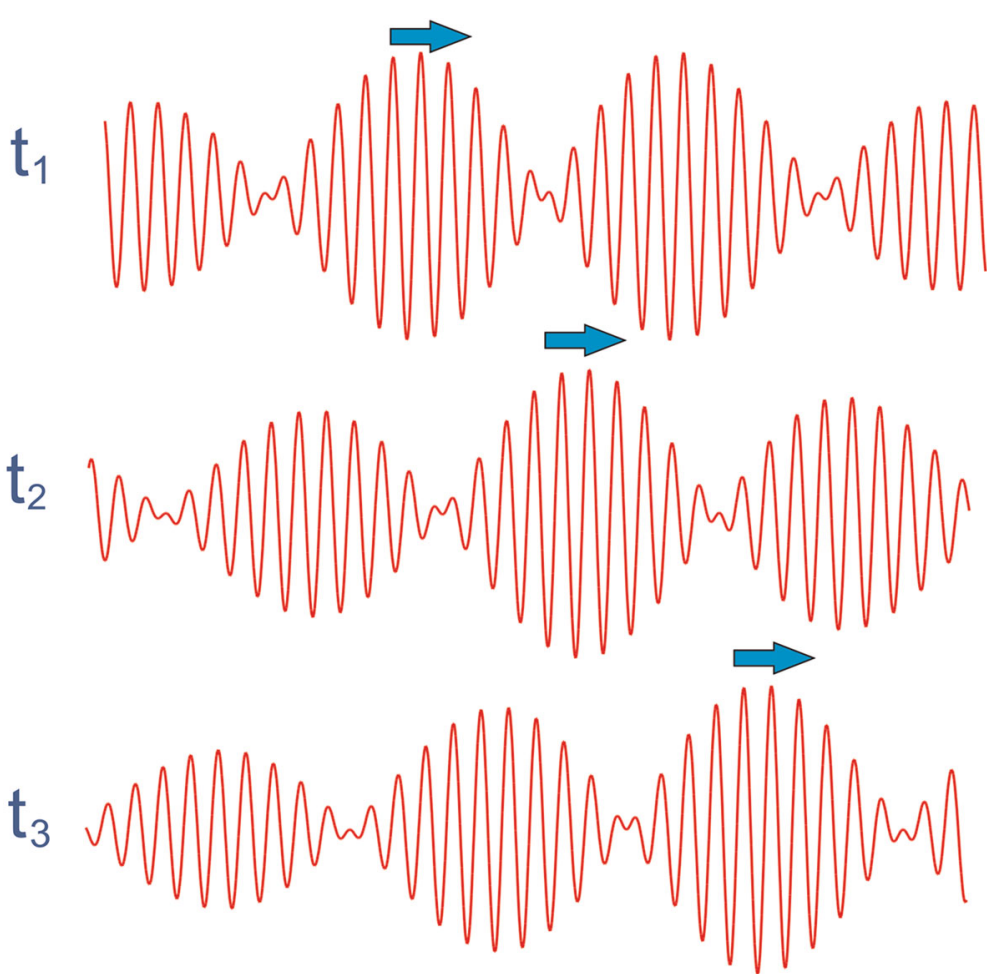

Fig. 2 Amplitudes (indicative only) at times $t_{1}<t_{2}<t_{3}$ for a wave of the kind shown in Fig. 1b. As shown by the arrows, the modulation (the de Broglie wave) develops through the carrier wave. It moves at the superluminal velocity $c^{2} / v$, while the carrier wave moves at velocity $v$, the classical velocity of the particle

$$
\Psi(x, t)=\left[e^{i\left(\omega_{1} t-\kappa_{1} x\right)}-e^{i\left(\omega_{2} t+\kappa_{2} x\right)}\right] / 2,
$$

which becomes, on taking real parts,

$$
\Psi(x, t)=\sin \left(\frac{\left(\omega_{1}-\omega_{2}\right)}{2} t-\frac{\left(\kappa_{1}+\kappa_{2}\right)}{2} x\right) \cos \left(\frac{\left(\omega_{1}+\omega_{2}\right)}{2} t-\frac{\left(\kappa_{1}-\kappa_{2}\right)}{2} x\right),
$$

which from relations (6) may also be written,

$$
\Psi(x, t)=\sin \gamma\left(\omega_{0} v t / c-\kappa_{0} x\right) \cos \gamma\left(\omega_{0} t-\kappa_{0} v x / c\right),
$$

and is a travelling wave of the kind illustrated in Fig. 2b. This one-dimensional travelling wave already displays features that will become more apparent when we consider the full three-dimensional travelling wave, namely a carrier wave of velocity $v$ (the first factor in Eq. 10) and a superluminal modulation (a beating) of velocity $c^{2} / v$ (the second factor). 5

\footnotetext{
5 The effect is strictly a "beating" between interfering waves of equal amplitude but differing characteristics.
} 


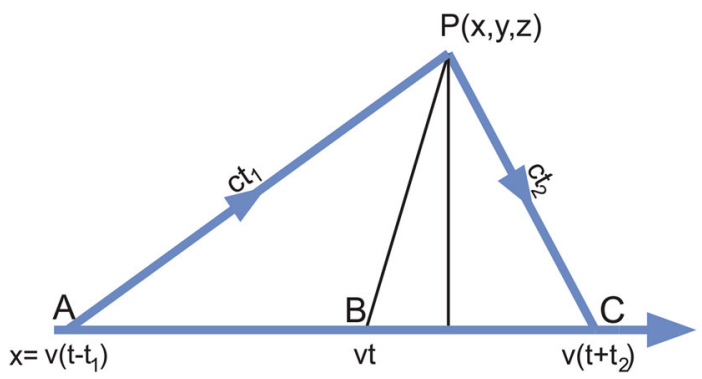

Fig. 3 With the centre of the wave of Fig. $1 \mathrm{~b}$ at $B$, interference occurs at $P(x, y, z)$ between the outgoing ray that left the wave centre at $A$ at the earlier time $t-t_{1}$, and the incoming ray that will not reach the centre until it is at $C$ at the later time $t+t_{2}$. The waveform thus constructed corresponds exactly to that obtained by Lorentz transforming the wave of Fig. 1a

The structure of the full model wave at velocity $v$ is now obtained by noticing that (as shown in Fig. 3) the amplitude of this wave at any point $P$ at time $t$, when the centre of the wave has reached $B$, results from the interference of the outgoing ray that left the particle centre when it was at $A$ at the earlier time $t-t_{1}$, with the incoming ray that will reach the particle centre when it is at $C$ at the later time $t+t_{2}$. (To an observer in the frame of the moving particle, the paths of these rays will appear to coincide, but they do not coincide in the laboratory frame).

In a more realistic model, phase might be expected to vary with angular displacement about the particle centre, but in this simple model we have supposed that all rays passing through the centre are of the same phase. Thus, from Eq. (9) for the one dimensional case,

$$
\begin{aligned}
& \Psi_{A}\left(t-t_{1}\right)=\exp i\left[\frac{\omega_{1}+\omega_{2}}{2}\left(1-\frac{v^{2}}{c^{2}}\right)\left(t-t_{1}\right)\right], \text { and } \\
& \Psi_{C}\left(t+t_{2}\right)=\exp i\left[\frac{\omega_{1}+\omega_{2}}{2}\left(1-\frac{v^{2}}{c^{2}}\right)\left(t+t_{2}\right)\right],
\end{aligned}
$$

(where the arguments of $\Psi_{A}$ and $\Psi_{C}$ are the phases at $A$ and $C$ respectively).

We now need $t_{1}$ and $t_{2}$. From Fig. 3,

$$
c^{2} t_{1}^{2}=\left[(x-v)\left(t-t_{1}\right)\right]^{2}+y^{2}+z^{2},
$$

from which,

$$
t_{1}=\frac{\frac{v}{c^{2}}(x-v t)+\frac{1}{c} \sqrt{(x-v t)^{2}+\left(1-v^{2} / c^{2}\right)\left(y^{2}+z^{2}\right)}}{1-v^{2} / c^{2}},
$$

and similarly,

$$
t_{2}=\frac{-\frac{v}{c^{2}}(x-v t)+\frac{1}{c} \sqrt{(x-v t)^{2}+\left(1-v^{2} / c^{2}\right)\left(y^{2}+z^{2}\right)}}{1-v^{2} / c^{2}} .
$$


Composing Eqs. (11) and (12), and substituting for $t_{1}$ and $t_{2}$ from Eqs. (13) and (14) respectively, we obtain after some algebra the full travelling wave,

$$
\Psi(x, y, z, t)=\sin \kappa_{o} \sqrt{\gamma^{2}(x-v t)^{2}+y^{2}+z^{2}} \cos \gamma \omega_{o}\left(t-v x / c^{2}\right),
$$

which is sketched for a particle of relativistic velocity in Fig. 1b. The corresponding amplitudes of such a wave (in the $x$-direction) are of the general form suggested by Fig. 2.

Travelling wave (15) could have been obtained by simply Lorentz transforming model wave (4). In following the longer route we have not derived the LT, for we have assumed the Lorentz factor $\gamma$ (an assumption that will be examined in Sect. 8), but we have demonstrated the physical effect of the LT, not as Einstein did with reference to rigid rods and synchronous clocks, but as Lorentz might have done, from the underlying oscillatory nature of matter. In doing so we have of course had the advantage of a unified view of matter and radiation that was not available to Lorentz.

It is important to notice the composite form of the transformed wave (15). It has two factors. The first,

$$
\sin \kappa_{o} \sqrt{\gamma^{2}(x-v t)^{2}+y^{2}+z^{2}}
$$

is a form of carrier wave. It moves with velocity $v$, the classical velocity of the particle and, as suggested by the ellipses in Fig. 1b, describes the relativistically contracted ellipsoidal form of the moving particle as considered from the standpoint of an observer in the laboratory frame. It has the frequency $\omega_{o} / \gamma$. The second factor,

$$
\cos \gamma \omega_{o}\left(t-v x / c^{2}\right)
$$

is a plane wave of superluminal velocity $c^{2} / v$, the wave fronts of which are suggested by the vertical lines in Fig. 1b. This plane wave has the wave number and frequency of the de Broglie wave, and is indeed the de Broglie wave. But it is not here an independent wave but a modulation of the carrier wave (16), describing the dephasing of that wave (and thus the failure of simultaneity) in the direction of travel of the particle.

Travelling wave (15) displays all the effects predicted by SR-length contraction, time dilation and failure of simultaneity. If all matter changed between inertial frames in the same manner as our model particle, this would explain why Mary considered Buzz to have changed in accordance with the LT. But the transformation is here a change of wave structure, not of space, nor even yet of reference frame. There has been no opportunity, let alone necessity, for some additional transformation of "spacetime".

What, then, of Buzz's belief that it was Mary who changed. In its altered state, the model particle has become a somewhat untidy affair. But to an observer moving with the particle (such as Buzz), and transformed in like manner, the particle will seem to have its original form (4). Being composed of influences developing at the speed of light, this observer will also lack the means of discerning differences in his or her velocity relative to that of light. In whatever inertial frame the observer occupies, the covariance and group theoretic properties of the LT will guarantee the constancy of the observed speed of light and the laws of physics. It is these properties that ensure 
that if a preferred frame does exist (of which more will be said in Sect. 7) it is likely to remain undetected.

\section{The de Broglie Wave}

Once the de Broglie wave is seen as a modulation rather than an independent wave, several mysteries become resolved. As a modulation, its superluminal velocity is no longer that of energy transport and need not be explained away by the usual but awkward device of equating the velocity of the particle with the group velocity of a packet of such de Broglie waves. It is also only natural that the velocity of this modulation should increase as the particle slows, and become infinite as the particle comes to rest. At rest, the crests of the composite wave are no longer peaking in sequence, but in unison. Simultaneity has been restored, alignment of phase has become instantaneous, and the velocity of the modulation describing the progress of that alignment has thus become infinite. In effect the de Broglie wave now disappears.

Other difficulties are explained. It can be seen why the de Broglie wave cannot be fitted to electron orbits. It is not the superluminal modulation but the full composite wave that follows the orbital path. Yet it is the modulation that defines the phase lost by the electron in following that path, and it is thus the de Broglie wave number $\kappa_{d B}$ of Eq. (3) that appears in Bohr quantization conditions for stable orbits, which are of general form,

$$
\oint \kappa_{d B} d s=2 n \pi \quad(n=1,2,3, \ldots),
$$

a requirement that evidently ensures continuity in the wave structure generated by the orbit in question.

The Schrödinger equation (SE) is also based on the modulation rather than the full wave, thus explaining the difficulties that have been experienced in according physical significance to solutions of this equation. ${ }^{6}$ In constructing a wave equation that would have solutions consistent with the Planck-Einstein and de Broglie relations (Eqs. (2), (3) above), Schrödinger made the substitutions,

$$
\begin{aligned}
& p \rightarrow i \hbar \frac{\partial}{\partial x}, \text { and } \\
& E \rightarrow i \hbar \frac{\partial}{\partial t},
\end{aligned}
$$

in the non-relativistic equation of motion,

$$
E^{2}=\frac{p^{2}}{2}+V(\mathbf{r}, t)
$$

to obtain the non-relativistic SE,

\footnotetext{
6 Of Schrödinger's own difficulties in interpreting those solutions, see Dorling [23].
} 


$$
i \hbar \frac{\partial \psi}{\partial t}=-\frac{\hbar^{2}}{2 m} \nabla^{2} \psi+V(\mathbf{r}, t) \psi
$$

and likewise in the relativistic equation of motion to obtain the corresponding relativistic wave equation (now called the Klein-Gordon equation).

But as we have seen, Eqs. (2) and (3) define the wave characteristics, not of the full waveform, but of the de Broglie wave. Thus solutions to the SE, whether in relativistic or non-relativistic form, are not actual waves but distorted mappings of a modulation. These solutions are able to identify allowed energy levels only because each such solution is homologous to a set of quantization conditions, the continuity implied by those conditions being at the same time the continuity required for physically realistic solutions of the SE.

Considered as a modulation, it also becomes understandable, not only that the superluminal wave does not outrun the subluminal particle, but why the wave vector $\kappa_{d B}$ of this wave has a controlling influence on the optical properties of the particle. Any change in the trajectory of the particle will be accompanied by a rotation throughout space of the wave fronts of the de Broglie wave and, as the immediate consequence of that rotation, a rearrangement of phase (and simultaneity) throughout the entire composite wave structure. It is only necessary to assume that a scattered particle (for instance a diffracted particle) will prefer a trajectory that preserves its characteristic transverse wave form to see why the particle tends to adopt a path through the scattering medium in which the de Broglie wave recombines coherently, that is to say, by which its recombining parts interfere constructively rather than destructively.

\section{The Sufficiency of the Model}

It might be objected that without knowing all there is to know about the constituent particles and fundamental forces of Nature, we are no better equipped than was Lorentz to base the LT on the properties of matter. It might also be argued that what we have been assuming are waves are not waves at all but particles that display oscillatory characteristics, or if waves, not real waves, but the probabilistic waves of standard quantum mechanics (SQM).

The model assumes that a massive particle comprises influences of frequency $\omega_{o}$ moving to and from the particle centre at velocity $c$. That the interaction of a particle with its fellows can be expressed in terms of incoming and outgoing influences is obvious and well recognized. ${ }^{7}$ It is also apparent that, as assumed in Sect. 4, the natural frequency $\omega_{o}$, which becomes $\gamma \omega_{o}$ for the moving particle, is not the measure of some merely internal property of the particle. This is sufficiently evidenced by the de Broglie wave, which is itself of frequency $\gamma \omega_{o}$, and reveals its presence externally in diffraction, interference and quantization. Conservation then demands that energies passing to and from the particle be commensurate, and thus the standing wave (4).

\footnotetext{
7 See, for example, the discussions of advanced and retarded influences by Wheeler and Feynman [24,25]. Those papers concerned action at a distance but such incoming and outgoing influences are explicit in field theories.
} 
ESR assumes that all influences in Nature propagate at velocity $c$. If some particle or force were shown to respond at a velocity differing from $c$, this would constitute as much an exception to ESR as it would to LSR. ${ }^{8}$ Massive particles do not move at that velocity, but it is implicit in ESR that the influences by which these particles interact do develop between and through the particles at velocity $c$. There is an analogy here with refraction. From interference between the incident (free space) wave, and reradiation from moments induced by that wave, the transmitted wave acquires a phase velocity that may be greater or smaller than $c$. Nonetheless, any change in the wave develops through the medium at the velocity $c$.

Let us suppose that some particle, let us say a meson mediating the strong force, or perhaps we should say, a string linking quarks, were to respond at velocity $C$ (differing from $c$ ) to some change in the relationship between one nucleon and another. The composition of incoming and outgoing influences could then be expressed in the form of Eq. (8), that is as,

$$
\Psi(\mathbf{r}, t)=\left[e^{i\left(\omega_{1} t-\kappa_{1} \cdot \mathbf{r}\right)}-e^{i\left(\omega_{2} t+\kappa_{2} \cdot \mathbf{r}\right)}\right] / 2,
$$

(or if not in that form then in some superposition of waves of that form), where contrary to relations (7) above,

$$
\frac{\omega_{1}}{\kappa_{1}}=\frac{\omega_{2}}{\kappa_{2}}=C \neq c
$$

From the discussion of the LT in Sect. 4, it is thus apparent that on a change of inertial frame, this supposed meson or string would suffer contraction, dilation and a changed simultaneity to a degree differing from that experienced by other matter, so that contrary to ESR (and as will be discussed in Sect. 8), the laws of physics would not then be the same in all inertial frames.

Once it is accepted that in its rest frame a particle comprises, or is at the focus of, influences oscillating at the natural frequency $\omega_{0}$ and moving inwardly and outwardly at velocity $c$, the exact nature of those influences becomes of no consequence to the demonstration in Sect. 4. These influences could be waves or fields, real, virtual or probabilistic, continuous or particulate. They might be concentrated upon some additional central body, but if having spatial extension, the material of that body would be of such a nature that influences propagating through the material did so at velocity $c$. Whatever the case, the moving "wave" will display the contraction and dilation described by the LT, and be subject to a modulation consistent with the predicted loss of simultaneity.

The plausibility of this model is evidenced by the physically consistent provenance it provides for the de Broglie wave. As to the infinity at the core of the model, it must be supposed that any viable wave form would exhibit some asymmetry avoiding such a singularity.

\footnotetext{
${ }^{8}$ We do not ignore the nonlocality implied by the formalism of SQM or the apparent confirmation of that nonlocality in dynamic Bell's experiments, notably those of Aspect et al. [26] and Weihs et al. [27]. We are content to rely (here at least) on the "peaceful coexistence" between SR and SQM declared by Shimony [28] from the circumstance that the nonlocality claimed does not permit superluminal signalling.
} 


\section{A Preferred Frame?}

We have supposed, with Lorentz, a preferred frame of reference- - the frame in which the speed of light is not only observed to be, but in fact is, the same in all directions. If, as seems the case, this frame is undetectable, it might be argued from considerations of economy that it should play no part in the description of SR. Einstein stated:

The introduction of a luminiferous aether will prove to be superfluous inasmuch as the view here to be developed will not require an "absolutely stationary space" provided with special properties [1].

Yet space does have at least one "special property" essential to ESR and assumed implicitly by Einstein. Unlike projectiles and massive particles, one photon never overtakes another. Photons pass through space at a common rate of progress, and their passage is thus similar in this respect to the propagation of a wave through an elastic medium, where the velocity of the disturbance is determined only by the nature of the wave and the properties of the medium.

Einstein suggested that the photon must be an "autonomous entity" requiring no supporting medium (see Kostro [29, pp. 37 and 94]), but it might be asked why such an unconstrained object should confine itself to a particular rate of progress. Moreover, photons not only propagate as waves, they have the oscillatory characteristics of waves. So too does matter. In the absence of some more plausible explanation, there would seem ample grounds for suspecting the light-carrying medium supposed by Lorentz.

Following the completion of GR, Einstein relaxed his stance against the aether, but not to the extent of admitting that such a medium could constitute a preferred frame (see generally Kostro [29]). Yet the argument for a preferred frame seems even stronger than that for an aether. It may be possible to imagine some reason other than a light-carrying medium for the photon's fixed rate of progress, perhaps the involvement in some way of the surrounding Universe. But it is the fixed rate of progress, not how it is enforced, that implies the existence of a frame of reference.

The argument becomes particularly insistent once the LT is explained from the wave characteristics of matter. There are practical and conceptual difficulties in presenting a wave based explanation of the LT without supposing such a frame. Consider, for instance, the frequencies $\omega_{1}$ and $\omega_{2}$ of Eq. (7). No matter how asymmetrical the Universe might be, it must be possible at least in principle to attain a velocity in any direction we choose for which one of these frequencies, $\left(\omega_{1}\right.$ or $\left.\omega_{2}\right)$ is the greater, and in the opposite direction for which the other is the greater, so that between these velocities, there must exist an inertial frame in which $\omega_{1}=\omega_{2}=\omega_{0}$. There must therefore exist, at least locally, a preferred frame.

These arguments appear as applicable in GR as in SR. However, in GR, the distribution of matter and radiation now hints at the location of such a frame. It would be the frame of the galaxies and of the cosmic microwave background - the frame from which spacetime itself is spatially isotropic - as is nearly so from the frame of our own local system.

Nor is the covariance of GR (general covariance) as convincingly agnostic of the existence and location of this frame as the LT of the special theory. It was objected by 
Kretschmann [30] in the first days of GR that by the sufficient exercise of mathematical ingenuity any equation of physics might be expressed in covariant form. Kretschmann thus argued that neither a relativity principle nor the primacy of Einstein's theory could be established by covariance per se. ${ }^{9}$ Einstein [34] conceded the point, whilst arguing for the superiority of his equation on the basis of simplicity. And he could also have cited of course the compelling utility and elegance of a manifest covariance.

Yet any inference from covariance that there is no preferred frame is weakened in GR by the existence of a gauge freedom allowing a choice of coordinates adapted to the symmetries of the problem, and thus in effect, to the curvature of the metric. The very notion of curvature implies a centre of curvature, and it becomes possible to think of the curvature tensor of the Universe at large as suggesting, mysterious though the concept may be, a preferred basis for the consideration of the curvature of the Universe itself.

Further arguments for a preferred frame have emerged that could not have been guessed at by Lorentz or Poincaré. It has been argued that such a frame is implied by the assumption, now orthodox, that the vacuum (the modern aether) is the seat of the zero point energies of the modes of the fields. This has suggested in turn the possibility of frame-dependent effects in the extreme and perhaps pathological situation at the event horizon of a black hole (see Winterberg [35]). There is also the hypothesized (and, it has been argued, detectable) Unruh-Davies effect, according to which an observer, who is accelerating with respect to the vacuum, experiences a thermal bath and consequent temperature rise from radiative effects (see, for example, Crispino et al. [36]).

However, it is not essential to the case for Lorentzian relativity presented here that this preferred frame have any particular location or that it be capable of detection. Indeed, there is reason to believe, as the next section may show, that this frame lies beyond all possibility of detection. But once the LT is explained, not from a change in the properties of space, but from changes in the wave-constituted material occupying that space, there must be in consequence one particular frame in which the underlying waves develop at the same speed in every direction.

If undetectable, this frame can play no essential role in the mathematical description of SR or GR. But with due respect to the friar of Occam, the dismissal of this frame as "superfluous" has had the effect of discouraging, if not entirely suppressing, lines of enquiry that imply its existence.

\section{Lorentz Invariance}

While there would seem little doubt that the laws of physics are the same for all observers, one might ask why this should be so. In the context of ESR this satisfying situation would seem fortuitous. However, a consideration of the wave nature of matter may suggest at least a partial explanation for this invariance.

\footnotetext{
${ }_{9}$ Kretschmann's objection has been much discussed, see for instance Norton [31], Dieks [32], and Giovanelli [33].
} 
Notice firstly the unusual effects that would result if the LT were not exactly as it is, for instance if the Lorentz factor $\gamma$ was not $\left(1-v^{2} / c^{2}\right)^{-1 / 2}$ but had some other value $\gamma^{a}$ ( $a$ being a real number other than unity). Consistently with relations (7), a wave travelling at any desired velocity $v$ could still be constructed, but as in the case of $a=0$, which is the Galilean transformation, the preferred frame would then be detectable. In the Galilean case, longitudinal contraction is replaced by transverse contraction that increases with acceleration away from the preferred frame. For other values of $a$, contraction or expansion (both longitudinal and transverse), time dilation and simultaneity would be observed to differ according to whether velocity were increased or decreased with respect to the preferred frame. The laws of physics would not then be invariant, and the Universe would be rather more curious and less elegant than it is. But that alone does not explain why it is not so.

Consider now, however, the stability of matter. The elementary particles exist in a limited variety of precisely defined characteristic forms. Why that should be so is not apparent, but what is apparent is that they are constrained by those forms and by their wave-like nature to combine in a limited number of ways, as is well illustrated by the Bohr conditions (18). Even in matter that we would think of as stationary, underlying microprocesses are occurring at relativistic speeds, as illustrated well enough again by the Bohr orbits. For these processes to remain undisturbed by a change of inertial frame, dynamic relationships between particles must be preserved, including for instance relative velocities, accelerations, masses and polarizations. In other words, the laws of physics must be the same for all inertial observers, and this is so only at $a=1$.

Considered in this way, Lorentz invariance is not the fortuitous cause, but rather the inevitable effect, of the manner in which the constituent elements of matter must persist and combine. It is not then the metric that determines how matter transforms, but the stability of matter that determines the LT and the observed (Minkowski) metric. Of any pre-existing or primordial metric little can be said, except that the simplest possible metric, the Euclidean, was that assumed by Lorentz.

\section{Summary}

The effects described by the LT can be explained in their entirety by changes occurring in matter as it changes inertial frame. This is not to suggest that the LT does not describe a transformation of space and time. But what the LT describes are changes in what is observed, and in the Lorentzian approach offered here, what is observed by an accelerated observer is essentially an illusion induced by changes in that observer.

This view relies crucially on the conclusion reached in Sect. 1 that the LT does not involve actual physical change in the properties of space. But once that conclusion is reached, it becomes apparent that there is something elusive in Einstein's theory, and that it is the Lorentzian approach that better explains the origin of the contraction, dilation and loss of simultaneity described by the LT.

Once the LT is explained from the wave characteristic of matter a good deal else becomes apparent. The de Broglie wave is seen to be a modulation rather than an independent wave, thus explaining the superluminality of this wave, its significance in 
the Schrödinger equation, and its roles in determining the optical properties of matter and the dephasing that underlies the relativity of simultaneity.

Einstein's bold assertion that the laws of physics must be the same for all observers revealed the elegance of SR and something indeed of the elegance of the Universe itself. It is suggested nonetheless that it is a Lorentzian approach that will provide the deeper understanding of the physical meaning of SR.

Acknowledgments The author thanks Will Nelson and Larry Hoffman for encouragement and helpful suggestions, and the anonymous reviewers for drawing attention to relevant and interesting issues arising in the context of general relativity.

Open Access This article is distributed under the terms of the Creative Commons Attribution License which permits any use, distribution, and reproduction in any medium, provided the original author(s) and the source are credited.

\section{References}

1. Einstein, A.: Zur elektrodynamik bewegter korper. Ann. Phys. 17, 891 (1905). (English trans.: On the electrodynamics of moving bodies. In: Lorentz, H. A., Einstein, A., Minkowski, H., Weyl, H.: The Principle of Relativity, Methuen, London, 1923)

2. Minkowski, H.: Raum und Zeit. Phys. Zeits. 10, 104 (1909). (English trans.: Space and time. In: Lorentz, H. A., Einstein, A., Minkowski, H., Weyl, H.: The Principle of Relativity, Methuen, London, 1923)

3. Rindler, W.: Relativity, Special, General, and Cosmological, 2nd edn. Oxford University Press, Oxford (2006)

4. Bell, J.S.: How to teach Special Relativity. Prog. Sci. Cult. 1, 2 (1976). (Reprinted in: Speakable and Unspeakable in Quantum Mechanics. Revised edn. Cambridge University Press, Cambridge, 2004)

5. Brown, H.S., Pooley, O.: Minkowski space-time: a glorious non-entity. In: Dieks, D. (ed.) The Ontology of Spacetime. Elsevier, Amsterdam (2006)

6. Brown, H.S.: Physical Relativity. Oxford University Press, Oxford (2005)

7. Miller, D.J.: A constructive approach to the special theory of relativity. Am. J. Phys. 78, 633 (2010)

8. Nelson, W.M.: A wave-centric view of special relativity. arXiv:1305.3022 [physics.class-ph] (2013)

9. de Broglie, L.: Recherches sur la thé orie des quanta, PhD Thesis. Ann. de Phys. 10 3, 22 (1925). (English trans. Researches on the quantum theory. In: Ann. Fond. Louis de Broglie 17, 92, 1992)

10. FitzGerald, G.F.: The ether and the earth's atmosphere. Science 13, 390 (1889)

11. Lorentz, H.A.: De relatieve beweging van de aarde en den aether. Zittingsverlag Akad. v. Wet. 1, 74 (1892). (English trans. The relative motion of the earth and the aether. In: Zeeman, P., Fokker, A.D. (eds.) Collected Papers. Nijhoff, The Hague, 1937)

12. Heaviside, O.: The electromagnetic effects of a moving charge. The Electrician 22, 147 (1888)

13. Larmor, J.: Aether and Matter. Cambridge University Press, Cambridge (1900)

14. Poincaré, H.: Sur la dynamique de l'électron. Rendiconti del Circolo matematico di Palermo 21, 129 (1906)

15. Reignier, J.: The birth of special relativity-One more essay on the subject. arXiv:physics/0008229 (2000).

16. Zahar, E.G.: Poincaré's independent discovery of the relativity principle. Fundam. Sci. 4, 147 (1983)

17. Lorentz, H.A.: The Theory of Electrons. Teubner, Leipzig (1916)

18. Wolff, M.: Fundamental laws, microphysics and cosmology. Phys Essays 6, 181 (1993)

19. de la Peña, L., Cetto, A.M.: The Quantum Dice: an Introduction to Stochastic Electrodynamics. Kluwer Academic, Dordrecht (1996)

20. Masreliez, C.J.: The Expanding Spacetime Theory. Nu, Corvallis (2000)

21. Baylis, W.E.: de Broglie waves as an effect of clock synchronization. Can. J. Phys. 85, 1317 (2007)

22. Keilman, Y.: Classical interpretation of de Broglie's waves. http://gsjournal.net. Accessed 2 Dec 2012

23. Dorling, J.: Schrödinger's original interpretation of the Schrödinger equation: a rescue attempt. In: Kilmister, C. (ed.) Schrödinger, centenary celebration of a polymath. Cambridge University Press, Cambridge (1987) 
24. Wheeler, J.A., Feynman, R.P.: Interaction with the absorber as the mechanism of radiation. Rev. Mod. Phys. 17, 157 (1945)

25. Wheeler, J.A., Feynman, R.P.: Classical electrodynamics in terms of direct interparticle action. Rev. Mod. Phys. 21, 425 (1949)

26. Aspect, A., Dalibard, J., Roger, G.: Experimental test of Bell's inequalities using time-varying analyzers. Phys. Rev. Lett. 49, 1804 (1982)

27. Weihs, G., Jennewein, T., Simon, C., Weinfurter, H., Zeilinger, A.: Violation of Bell's inequality under strict Einstein locality conditions. Phys. Rev. Lett. 81, 5039 (1998)

28. Shimony, A., et al.: Controllable and uncontrollable non-locality. In: Kamefuchi, S. (ed.) Foundations of Quantum Mechanics in the Light of New Technology, p. 225. Physical Society of Japan, Tokyo (1983)

29. Kostro, L.: Einstein and the Ether. Apeiron, Montreal (2000)

30. Kretschmann, E.: Über den physikalischen Sinn der Relativitätspostulate. A. Einsteins neue und seine ursprüngliche Relativitätstheorie. Ann. Phys. 53, 575 (1917)

31. Norton, J.D.: General covariance and the foundations of general relativity: eight decades of dispute. Rep. Prog. Phys. 56, 791 (1993)

32. Dieks, D.: Another look at general covariance and the equivalence of reference frames. Stud. Hist. Philos. Mod. Phys. 37, 174 (2006)

33. Giovanelli, M.: Erich Kretschmann as a proto-logical-empiricist: adventures and misadventures of the point-coincidence argument. Stud. Hist. Philos. Mod. Phys. 44, 155 (2013)

34. Einstein, A.: Prinzipielles zur allgemeinen relativit ätstheorie. Ann. Phys. 55, 240 (1918)

35. Winterberg, F.: Gamma ray bursters and Lorentzian relativity. Z. Naturforsch. 56A, 889 (2001)

36. Crispino, L.C.B., Higuchi, A., Matsas, G.E.A.: The Unruh effect and its applications. Rev. Mod. Phys. 80, 787 (2008) 